\title{
VARIACIÓN MORFOLÓGICA DEL GÉNERO PROCHNYANTHES (AGAVACEAE)
}

\author{
Arturo Castro-Castro, Aarón Rodríguez, Georgina Vargas-Amado y \\ RAYMUNDO RAMÍREZ-DELGADILLO \\ Universidad de Guadalajara, Departamento de Botánica y Zoología, \\ Apdo. postal 139, 45101 Zapopan, Jalisco, México. \\ arca68@hotmail.com
}

\section{RESUMEN}

Se analizó la variación morfológica del género Prochnyanthes, mediante el examen de 29 caracteres, en una muestra de 544 individuos de 19 localidades. Se aplicó el Análisis de Componentes Principales, el cual muestra los patrones de variación, analiza las similitudes de las unidades de clasificación e identifica grupos. Con base en la evidencia morfológica, se amplió la descripción de $P$. mexicana y se logró reconocer dos fenotipos, considerándolos como extremos de su variación. El tamaño y el color del perianto así como la longitud de los pedicelos no mostraron la suficiente variación para diferenciar grupos y en consecuencia se reconoció a $P$. mexicana como la única especie del género.

Palabras clave: Agavaceae, análisis de componentes principales, morfología, Prochnyanthes.

\begin{abstract}
The morphologic variation of the genus Prochnyanthes was analyzed by means of the examination of 29 characters, in a sample of 544 individuals from 19 localities. The Principal Components Analysis was used, which shows the variation trends, analyses the similarities among the classification units and identifies groups. Based on the morphological evidence, it was possible to recognize two phenotypes, considered as extremes of a morphological cline. Therefore, the morphological species concept of P. mexicana was broaden to include all the variation observed. Finally, the size and color of the perianth and the length of the pedicels did not show enough variation to identify groups and consequently P. mexicana is the only recognized species of the genus.
\end{abstract}

Key words: Agavaceae, morphology, principal components analysis, Prochnyanthes. 


\section{INTRODUCCIÓN}

El género Prochnyanthes S. Watson es endémico de México. Sus componentes crecen a lo largo de la Sierra Madre Occidental y el Eje Volcánico Transversal en los estados de Aguascalientes, Durango, Guanajuato, Jalisco, Michoacán, Nayarit, Querétaro y Zacatecas (García-Mendoza y Galván, 1995). Habitan en laderas rocosas con vegetación de bosque de encino, pino y encino, pastizal y zonas de transición entre el encinar y el bosque tropical caducifolio, en altitudes de 1000 a $2700 \mathrm{~m}$. Los nombres comunes registrados para Prochnyanthes son amole, amolilla, huaco y lirio. El macerado del cormo y de las raíces se utiliza como sustituto del jabón e insecticida (Verhoek, 1978).

Las relaciones filogenéticas de Prochnyanhtes han sido analizadas pero no existe hasta ahora un acuerdo al respecto. Bogler y Simpson (1996) mostraron la estrecha relación entre los géneros Manfreda Salisb., Polianthes L. y Prochnyanthes, considerándolos como un clado independiente de Agave L. Más aún, Thiede y Eggli (1999), con base en datos moleculares y morfológicos (Bogler et al., 1995; Bogler y Simpson, 1995, 1996; Clary y Simpson, 1995; Hernández, 1995), incluyeron a los géneros Manfreda, Polianthes y Prochnyanthes dentro de Agave, reconociendo el epíteto Agave bulliana (Baker) Thiede \& Eggli, bajo el cual se incluye a Prochnyanthes mexicana.

Los límites interespecíficos dentro del género son poco claros. Algunos autores consideran que se trata de una sola especie morfológicamente muy variable (Verhoek-Williams, 1975; McVaugh, 1989; Espejo-Serna y López-Ferrari, 1993; Williams, 1998). Otros reconocen más y han descrito a las especies: P. viridescens S. Watson (1887), P. bulliana Baker (1895) y P. mexicana (Zucc.) Rose (1903) como taxa separados. Los caracteres utilizados para su delimitación son la longitud del pedicelo y el tamaño del perianto; sin embargo, estos rasgos son los que muestran mayor variación entre individuos de una población e incluso en un mismo individuo pero no distinguen a poblaciones.

Prochnyanthes bulliana fue diferenciada de $P$. viridescens con base en sus pedicelos cortos y flores púrpura-verdosas. Una segunda descripción indica que $P$. bulliana presenta flores sésiles con la parte externa del perianto de color verde-pardo y el interior amarillento (Baker 1884, 1895). Prochnynahtes mexicana fue definida como planta con flores sésiles de perianto más largo y delgado que el de P. bulliana. De acuerdo con el protólogo de $P$. viridescens, ésta presenta flores amarillo-verdosas con tintes pardos y pedicelos ascendentes de 2.5-4 cm de longitud (Verhoek-Williams 1975; Cuadro 1). 
Cuadro 1. Principales caracteres utilizados en la delimitación de especies en Prochnyanthes.

\begin{tabular}{|c|c|c|c|}
\hline & $\begin{array}{l}\text { P. viridescens } \\
\text { S. Watson, } 1887\end{array}$ & $\begin{array}{l}\text { P. bulliana } \\
\text { J. G. Baker, } 1895\end{array}$ & $\begin{array}{l}\text { P. mexicana } \\
\text { J. N. Rose, } 1903\end{array}$ \\
\hline Pedicelos & $\begin{array}{l}\text { ascendentes, de } 2.5 \text {-4 } \\
\text { cm de longitud }\end{array}$ & muy cortos & ausentes \\
\hline Perianto & $\begin{array}{l}\text { amarillo-verdoso a } \\
\text { pardo, de } 2.4 \mathrm{~cm} \text { de } \\
\text { longitud, tubo de } 0.6 \\
\text { cm longitud }\end{array}$ & $\begin{array}{l}\text { externamente púrpura-verdoso } \\
\text { con extremos blanquecinos e } \\
\text { internamente amarillento, de } 3 \\
\mathrm{~cm} \text { de longitud, tubo de } 0.4 \mathrm{~cm} \\
\text { de diámetro, boca del perianto } \\
\text { de } 0.8 \mathrm{~cm} \text { de diámetro }\end{array}$ & $\begin{array}{l}\text { más largo y delgado } \\
\text { que en } P \text {. bulliana }\end{array}$ \\
\hline
\end{tabular}

El propósito del presente trabajo fue estudiar estadísticamente la variación morfológica en Prochnyanthes, mediante la aplicación del Análisis de Componentes Principales (ACP) que permite analizar la matriz de correlaciones y transformar un grupo de variables relacionadas en un conjunto menor de variables independientes entre sí llamadas componentes principales. De esta manera, se consideran a la vez varias características, resolviendo las dimensiones latentes en los datos (Lamboy, 1990; Torres, 2000), y se analizan los patrones de variación.

\section{MÉTODOS}

Se estudiaron 19 poblaciones localizadas en los estados de Aguascalientes, Durango, Guanajuato, Jalisco, Michoacán, Nayarit, Querétaro y Zacatecas (Apéndice 1). El criterio para la selección de los sitios de colecta se basó en ejemplares de herbario que durante la revisión mostraron características interesantes para el análisis y que cubrieron la mayor parte del área de distribución. También se consideraron las sugerencias de otros botánicos, lo reportado en la literatura botánica y la experiencia personal producto de colectas previas. Asimismo, se puso interés especial en las localidades tipo (Zuccarini, 1837; Baker, 1884, 1895; Watson, 1887; Rose, 1903; McVaugh, 1989).

La consulta de herbarios y recolección de material botánico se realizó de agosto a octubre de 2005. Se revisaron 95 ejemplares botánicos de los siguientes herbarios: Herbario de la Universidad de Guadalajara (IBUG), Herbario del Instituto de Ecología A.C., Centro Regional del Bajío (IEB), Herbario del Centro Inter- 
disciplinario para el Desarrollo Integral Regional-Durango (CIIDIR), Herbario del Instituto de Biología de la Universidad Nacional Autónoma de México (MEXU) y el Herbario de la Universidad Autónoma de Querétaro (QMEX). Estos ejemplares no se analizaron porque no se apreciaban en ellos todas las características estudiadas (Apéndice 2).

Se examinaron 30 individuos vivos en estado reproductivo por población. La única excepción fue la del estado de Querétaro, en el cual sólo fue posible estudiar cuatro plantas (Apéndice 1). Se evaluaron 29 caracteres (Fig. 1; Cuadro 2). Las características florales se obtuvieron del par de flores con el estilo maduro al momento de la medición. El tamaño de la muestra consistió de 544 ejemplares. La matriz básica de datos está disponible a través del primer autor. Se colectaron cinco individuos por población y se distribuyeron a los siguientes herbarios: ENCB, IBUG, IEB, MEXU y XAL.

El ACP se ejecutó a través del programa de cómputo SPSS 9.0 (Anónimo, 1999) y se realizaron dos análisis. En el primero se consideraron las 29 variables y se encontró que ocho de ellas: longitud de la bráctea del pedúnculo, longitud de la antera, ancho de la antera, longitud del estilo, ancho del estilo, ancho de la bráctea del pedúnculo, ancho de la bráctea del raquis y ancho del ovario, presentaron coeficientes de correlación bajos y aportaron poca variación al modelo. En consecuencia, se eliminaron de subsecuentes análisis. Asimismo, la longitud del pedúnculo y la longitud del raquis fueron eliminadas por ser redundantes, debido a que se consideraron variables correlacionadas lógicamente con la longitud de la inflorescencia, además de mostrar bajo aporte de variación.

Con base en los resultados del primer ACP, se elaboraron variables compuestas para la ejecución del segundo análisis. Estas fueron las siguientes proporciones: 1) largo/ancho del perianto (forma del perianto), 2) largo/ancho de las hojas (forma de las hojas), 3) largo/ancho de las anteras (forma de las anteras), y 4) largo/ancho del pedicelo (forma del pedicelo). La forma de la hoja y pedicelo, así como la longitud del perianto y filamento aportaron poca variación al modelo, sin embargo se decidió conservarlos para el análisis debido a su supuesto valor en el reconocimiento de las especies de Prochnyanthes (Cuadro 1).

\section{RESULTADOS}

El análisis mostró siete componentes principales que contienen $77.9 \%$ de la variación total (Cuadro 3). La extracción se realizó con base en el criterio de raíz 
Cuadro 2. Descripción de caracteres analizados.

\begin{tabular}{|c|c|c|}
\hline Carácter & Abreviatura & Descripción \\
\hline $\begin{array}{l}\text { Largo y ancho de las } \\
\text { hojas }\end{array}$ & $\begin{array}{l}\text { HL (largo) HA } \\
\text { (ancho) }\end{array}$ & $\begin{array}{l}\text { El largo se midió desde la base de la hoja en donde inicia el } \\
\text { cormo hasta el ápice. El ancho se tomó en la parte más amplia. }\end{array}$ \\
\hline $\begin{array}{l}\text { Longitud de la } \\
\text { inflorescencia }\end{array}$ & I & $\begin{array}{l}\text { Desde su inserción en el cormo hasta el ápice en el último } \\
\text { nudo floral. }\end{array}$ \\
\hline Largo del pedúnculo & $\mathrm{P}$ & $\begin{array}{l}\text { Desde la inserción de la inflorescencia en el cormo hasta } \\
\text { el primer nudo floral. }\end{array}$ \\
\hline Largo del raquis & $\mathrm{R}$ & $\begin{array}{l}\text { Desde el primer nudo floral de la inflorescencia hasta su } \\
\text { ápice. }\end{array}$ \\
\hline $\begin{array}{l}\text { Largo y ancho de la } \\
\text { bráctea del pedúnculo }\end{array}$ & $\begin{array}{l}\text { BPL (largo) } \\
\text { BPA (ancho) }\end{array}$ & $\begin{array}{l}\text { Última bráctea del pedúnculo en sus porciones más largas } \\
\text { y anchas. }\end{array}$ \\
\hline $\begin{array}{l}\text { Largo y ancho de la } \\
\text { bráctea del raquis }\end{array}$ & $\begin{array}{l}\text { BRL (largo) } \\
\text { BRA (ancho) }\end{array}$ & $\begin{array}{l}\text { Primera bráctea del raquis en sus porciones más largas y } \\
\text { anchas. }\end{array}$ \\
\hline $\begin{array}{l}\text { Largo y ancho del } \\
\text { pedicelo }\end{array}$ & $\begin{array}{l}\text { PeL (largo) } \\
\text { PeA (ancho) }\end{array}$ & $\begin{array}{l}\text { Largo: desde su origen en el raquis de la inflorescencia } \\
\text { hasta la base del ovario. Ancho: en su base. }\end{array}$ \\
\hline $\begin{array}{l}\text { Largo y ancho de la } \\
\text { bractéola }\end{array}$ & $\begin{array}{l}\text { BracL (largo) } \\
\text { BracA (ancho) }\end{array}$ & $\begin{array}{l}\text { Largo: desde su inserción en la base del pedicelo hasta su } \\
\text { ápice. Ancho: porción más amplia de su base. }\end{array}$ \\
\hline $\begin{array}{l}\text { Largo y ancho de la } \\
\text { porción amplia del } \\
\text { perianto }\end{array}$ & $\begin{array}{l}\text { PerL (largo) } \\
\text { PerA (ancho) }\end{array}$ & $\begin{array}{l}\text { Representa la porción amplia del perianto junto con sus ló- } \\
\text { bulos. Largo: desde su unión a la porción estrecha del tubo } \\
\text { hasta el ápice de los lóbulos extendidos. Ancho: diámetro } \\
\text { del perianto medido en el ápice de los lóbulos extendidos. }\end{array}$ \\
\hline $\begin{array}{l}\text { Largo y ancho del lóbulo } \\
\text { externo del perianto }\end{array}$ & $\begin{array}{l}\text { LeL (largo) } \\
\text { LeA (ancho) }\end{array}$ & $\begin{array}{l}\text { Largo: desde su origen en la parte distal de la porción am- } \\
\text { plia del tubo del perianto hasta el ápice. Ancho: distancia } \\
\text { entre los bordes en su valor máximo. }\end{array}$ \\
\hline $\begin{array}{l}\text { Largo y ancho del lóbulo } \\
\text { interno del perianto }\end{array}$ & $\begin{array}{l}\text { LiL (largo) } \\
\text { LiA (ancho) }\end{array}$ & $\begin{array}{l}\text { Largo: desde su origen en la parte distal de la porción am- } \\
\text { plia del tubo del perianto hasta el ápice. Ancho: distancia } \\
\text { entre los bordes en su valor máximo. }\end{array}$ \\
\hline $\begin{array}{l}\text { Largo y ancho de la } \\
\text { porción estrecha del tubo } \\
\text { del perianto }\end{array}$ & $\begin{array}{l}\text { TPL (largo) } \\
\text { TPA (ancho) }\end{array}$ & $\begin{array}{l}\text { Representa la porción estrecha del tubo del perianto. Lar- } \\
\text { go: desde su base en la unión al ovario hasta la base de la } \\
\text { porción amplia del tubo del perianto. Ancho: diámetro en } \\
\text { su valor máximo. }\end{array}$ \\
\hline Largo del filamento & Fil & $\begin{array}{l}\text { Desde su inserción en el perianto hasta su unión a la an- } \\
\text { tera. }\end{array}$ \\
\hline $\begin{array}{l}\text { Largo y ancho de la } \\
\text { antera }\end{array}$ & $\begin{array}{l}\text { AntL (largo) } \\
\text { AntA (ancho) }\end{array}$ & $\begin{array}{l}\text { Largo: distancia entre los extremos longitudinales. An- } \\
\text { cho: valor máximo entre sus bordes. }\end{array}$ \\
\hline Ancho del estigma & $\mathrm{AE}$ & Valor máximo entre los extremos de los lóbulos. \\
\hline Largo y ancho del estilo & $\begin{array}{l}\text { EL (largo) } \\
\text { EA (ancho) }\end{array}$ & $\begin{array}{l}\text { Largo: desde su origen en el ovario hasta su terminación } \\
\text { en el estigma. Ancho: diámetro en su base. }\end{array}$ \\
\hline Largo y ancho del ovario & $\begin{array}{l}\text { OL (largo) } \\
\text { OA (ancho) }\end{array}$ & $\begin{array}{l}\text { Largo: desde su base e inserción al pedicelo hasta su unión } \\
\text { al estilo. Ancho: valor máximo del diámetro. }\end{array}$ \\
\hline
\end{tabular}




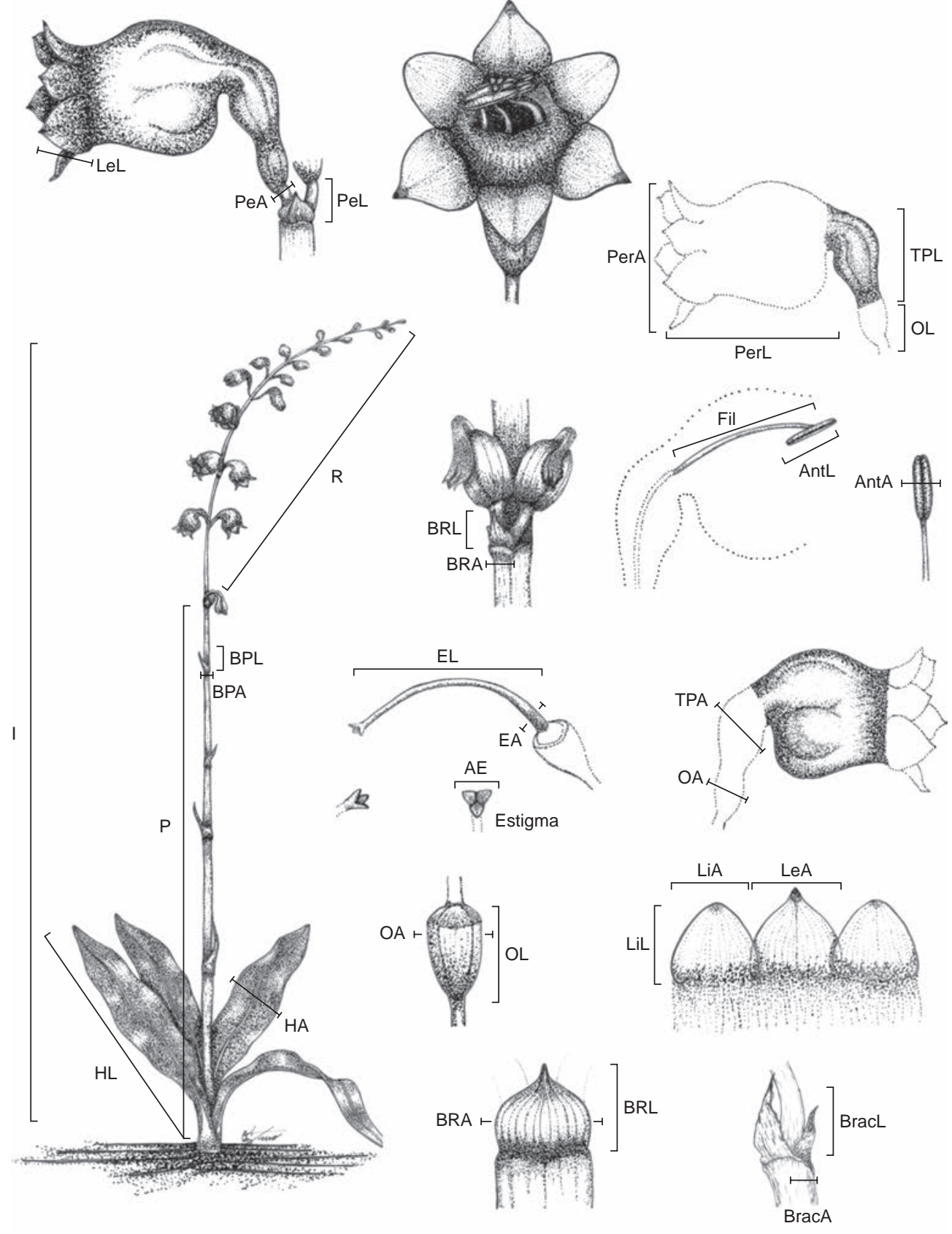

Fig. 1. Caracteres analizados, abreviaturas conforme a lo descrito en Cuadro 2. Ilustración: Osvaldo Zuno 2006. 
Cuadro 3. Extracción de componentes principales.

\begin{tabular}{cccc}
\hline Componente & Eigenvalor & \% de variación & \% acumulativo \\
\hline I & 8.8 & 38.5 & 38.5 \\
II & 2.0 & 8.7 & 47.2 \\
III & 1.9 & 8.3 & 55.6 \\
IV & 1.6 & 7.1 & 62.7 \\
V & 1.3 & 5.8 & 68.6 \\
VI & 1.1 & 4.8 & 73.5 \\
VII & 1.0 & 4.4 & 77.9 \\
\hline
\end{tabular}

latente (Crisci y López-Armengol, 1983; Hair et al., 1999). De las 23 variables estudiadas, 20 están contenidas en los tres primeros componentes: 15 en el principal, tres en el segundo y dos en el tercero (Cuadro 4).

Generalmente y con fines prácticos se utilizan e interpretan los tres primeros componentes que contienen la mayor variación, lo que reduce el número de dimensiones (Anónimo, 1999). En el presente estudio, la variación contenida en los tres primeros es de $55.6 \%$.

El componente principal I (CP I) acumuló 38.5\% de la variación. Éste tiene relación con la talla de la planta y la forma del perianto; aquellos individuos que tienen mayores dimensiones, tienden a presentar flores campanuladas (Fig. 2. C-L). En contraste, los de menor tamaño, propenden a producir flores tubulares (Fig. 2. A, B). Los caracterizados por tallas menores y flores tubulares (CP I menores a -1, CP II mayores a 0, Fig. 3) forman parte de las dos poblaciones del estado de Aguascalientes y de la correspondiente al Puerto la Campana, municipio de Atenguillo, Jalisco. En el otro extremo, las poblaciones con individuos grandes y de flores campanuladas son características del resto de la muestra y representan $77.9 \%$. Este último fenotipo concuerda con las descripciones realizadas por Baker (1884 y 1895), Watson (1887) y Rose (1903) y coincide con lo examinado por Verhoek-Williams (1975) y Williams (1998). La talla de la planta y la forma del perianto permiten el reconocimiento de dos morfos; sin embargo, sólo se pueden considerar extremos de variación de una sola especie, ya que de acuerdo con la gráfica de dispersión entre el CP I y el CP II, no cabe reconocer grupos independientes (Fig. 3).

En general, existe una alta relación morfológica entre los individuos analizados. No obstante, se observan extremos de la variación que corresponden a las dos 
Cuadro 4. Variación aportada por cada variable a los componentes principales (CP); los valores en negritas conforman el CP correspondiente.

\begin{tabular}{|c|c|c|c|}
\hline \multirow{2}{*}{ Caracteres } & \multicolumn{3}{|c|}{$\mathrm{CP}$} \\
\hline & I & II & III \\
\hline 1. longitud de la hoja & .644 & $1.317 \mathrm{E}-02$ & 4.782E-02 \\
\hline 2. ancho de la hoja & .396 & -.647 & .374 \\
\hline 3. largo/ancho de la hoja (forma de la hoja) & $-6.891 \mathrm{E}-03$ & .654 & -.322 \\
\hline 4. longitud de la inflorescencia & .543 & -.363 & .176 \\
\hline 5. longitud de la bráctea del raquis & .665 & $-7.075 \mathrm{E}-02$ & .259 \\
\hline 6. longitud del pedicelo & .639 & .368 & .399 \\
\hline 7. ancho del pedicelo & .666 & -.165 & -.342 \\
\hline 8. largo/ancho del pedicelo (forma del pedicelo) & .385 & .458 & .559 \\
\hline 9. longitud de la bractéola & .284 & .228 & .616 \\
\hline 10. ancho de la bractéola & 2.791E-02 & .130 & .521 \\
\hline 11. longitud del perianto & .498 & .405 & -.173 \\
\hline 12. ancho del perianto & .834 & -.100 & -.109 \\
\hline 13. largo/ancho del perianto (forma del perianto) & -.609 & .324 & 5.031E-03 \\
\hline 14. longitud del lóbulo externo & .887 & $6.587 \mathrm{E}-02$ & $-5.133 E-02$ \\
\hline 15. ancho del lóbulo externo & .902 & $-6.325 \mathrm{E}-02$ & -.144 \\
\hline 16. longitud del lóbulo interno & .886 & .111 & $-3.802 \mathrm{E}-02$ \\
\hline 17. ancho del lóbulo interno & .914 & $-6.904 \mathrm{E}-02$ & $-9.368 \mathrm{E}-02$ \\
\hline 18. longitud del tubo del perianto & .643 & $-1.346 \mathrm{E}-02$ & -.307 \\
\hline 19. ancho del tubo del perianto & .825 & $-6.651 \mathrm{E}-03$ & -.142 \\
\hline 20. longitud del filamento & .403 & .445 & 1.920E-02 \\
\hline 21. largo/ancho antera (forma de la antera) & -.263 & .277 & -.248 \\
\hline 22. ancho del estilo & .336 & 4.502E-03 & 7.999E-02 \\
\hline 23. longitud del ovario & .702 & .107 & -.271 \\
\hline
\end{tabular}

localidades del estado de Aguascalientes. Los ejemplares de San José de Gracia (AGSGracia) muestran caracteres intermedios entre los de Sierra Fría (AGSsp) y todos los demás, siendo este último sitio el que señaló mayor diferencia fenotípica (Fig. 2; Apéndice 1).

Las plantas estudiadas de Jalisco señalaron un amplio intervalo de variación morfológica y distribución espacial, relacionándose con el resto de los sujetos ana- 
Castro-Castro et al.: Variación morfológica del género Prochnyanthes (Agavaceae)
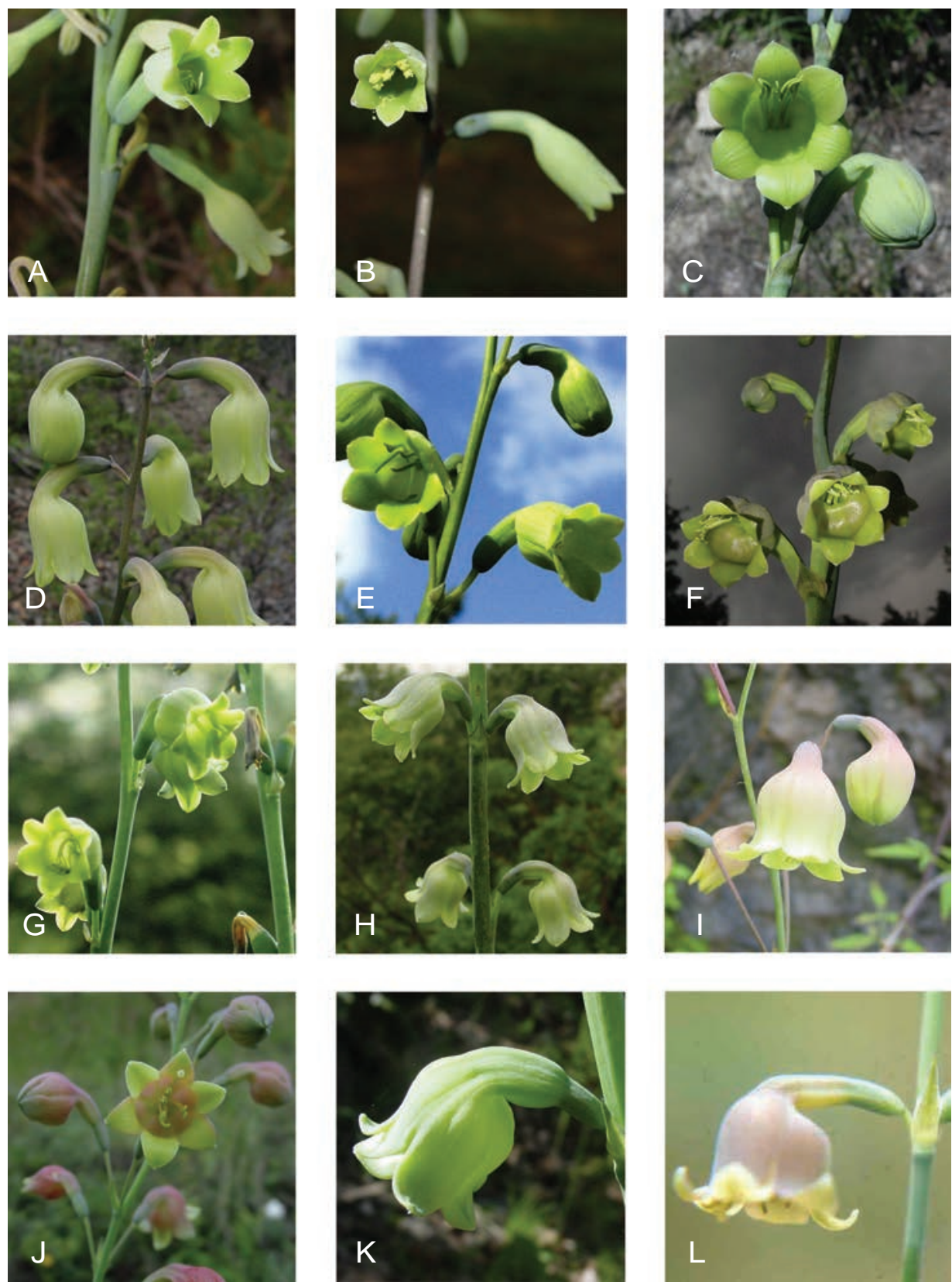

Fig. 2. Variación floral de Prochnyanthes. A:AGSGracia ; B: AGSsp; C: DGOsalt; D: DGOsuch; E: GTOJ.R; F: MICH; G: QRO; H: ZACFlor; I: JALAmec; J: JALpinar; K: ZACMor; L: JALTapalp. (Abreviaturas ver apéndice 1. Fotografias A, B, F y H por R. Ramírez-Delgadillo 2005, C, D, I, J, K y L por A. Rodríguez 2005 y, E y G por A. Castro-Castro 2005). 


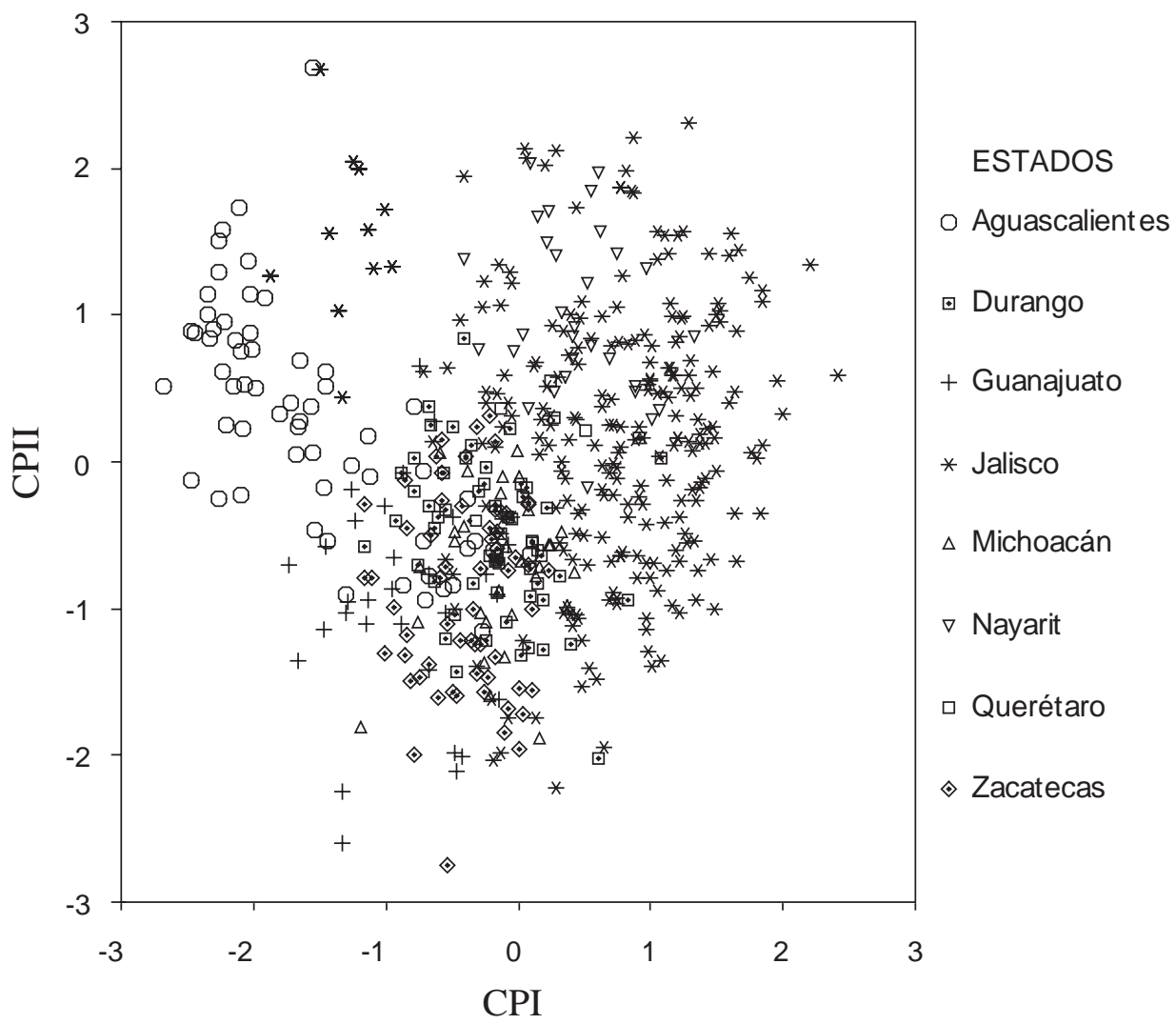

Fig. 3. Distribución de organismos analizados por muestras de cada estado con base en los componentes principales (CP) I y II; cada símbolo representa al menos un individuo.

lizados (Fig. 3, 4). Se observa una gran similitud de los organismos entre localidades, con una agrupación centralizada en torno a la localidad tipo de Prochnyanthes viridescens (JALR.Bco) y extremos de variación que corresponden a las poblaciones del Puerto la Campana (JALCampa), Ameca (JALAmec) y Tapalpa (JALTapalp; Apéndice 1).

El componente principal II (CP II) contiene $8.7 \%$ de la variación. Se relaciona negativamente con el ancho de la hoja y positivamente con la longitud del filamento y la forma de la misma. Es decir, a medida que aumenta el ancho de la hoja, ésta tiende a ser elíptica y se presentan flores con filamentos cortos. Sin embargo, tal variación es clinal, de modo que la forma foliar y el largo del filamento no permiten el reconocimiento de ningún fenotipo. 


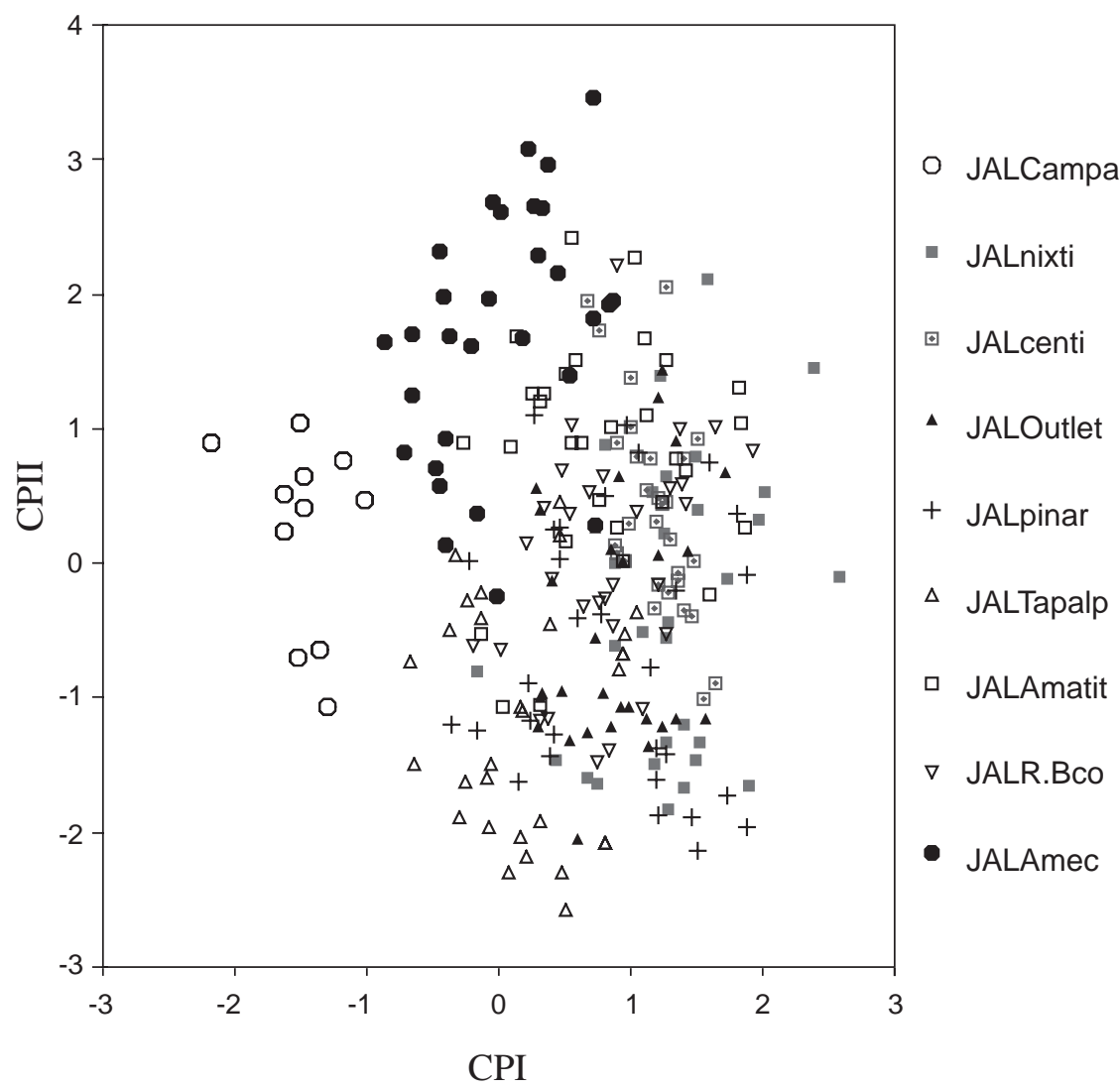

Fig. 4. Dispersión de organismos analizados en el estado de Jalisco con base en los componentes principales (CP) I y II; los símbolos representan al menos un individuo. Abreviaturas de acuerdo con el Apéndice 1.

El componente principal III (CP III) acumula $8.3 \%$ de la variación. Se relaciona positivamente con la forma del pedicelo y con la longitud de la bractéola. Por tradición, la longitud del pedicelo ha sido utilizada como un carácter útil en la delimitación de las especies (Baker, 1895; Watson, 1887; Rose, 1903). Sin embargo, en nuestro análisis la forma del pedicelo y el largo de la bractéola no permiten el reconocimiento de fenotipos. La figura 5 muestra la distribución espacial entre el CP II y el CP III. En su extremo superior se observan agrupados individuos con pedicelos largos (1.375-8.94 cm), los cuales corresponden a las localidades de Ameca y Amatitán en el estado de Jalisco, que se ajustan a la descripción de Prochnyanthes viridescens (Watson, 1887) y que proceden de sitios cercanos a la localidad tipo (Río 


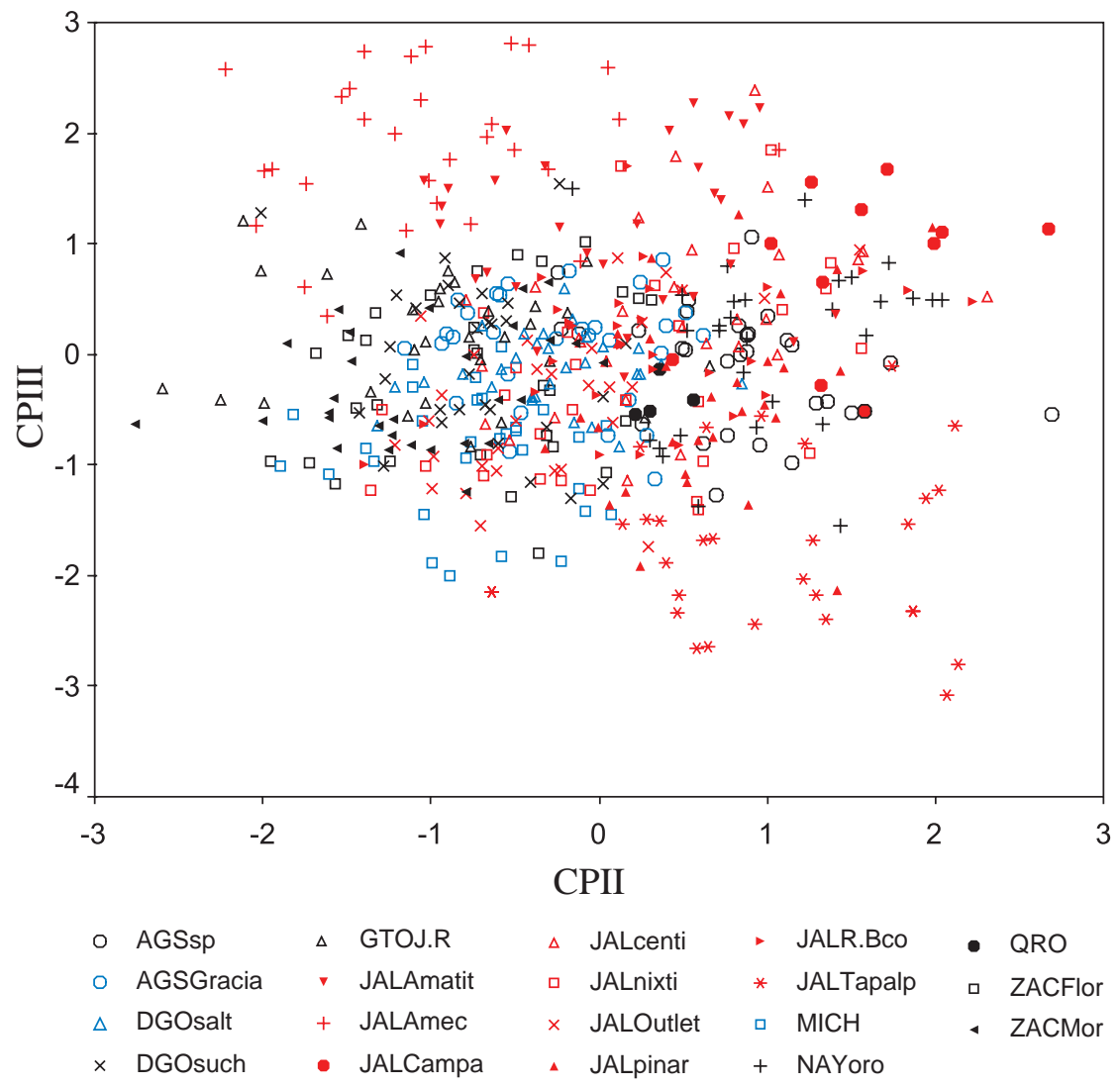

Fig. 5. Grafica de dispersión de los individuos por localidad analizada, con base en los componentes principales (CP) II y III. Donde cada símbolo representa al menos un individuo; abreviaturas conforme al Apéndice 1.

Blanco, Zapopan, Jalisco). Por su parte, los de Tapalpa, Jalisco, con pedicelos cortos (0.329-2.097 cm, Fig. 2 I, L), se asemejan mejor a lo referido por Baker (1895) y Rose (1903) (Cuadro 1).

La figura 6 exhibe las tendencias fenotípicas de los individuos en relación con su ubicación en los diferentes estados de la República. Se observa afinidad entre los organismos de los estados de Jalisco y Nayarit (tendencia A), que se caracterizan por presentar hojas elípticas y filamentos largos, respecto a aquellos de Zacatecas, Durango, Guanajuato y Michoacán (tendencia B), con características antagónicas. Por último, las poblaciones del estado de Aguascalientes (tendencia C, Fig. 2) se diferencian por tener tallas menores y flores tubulares. 


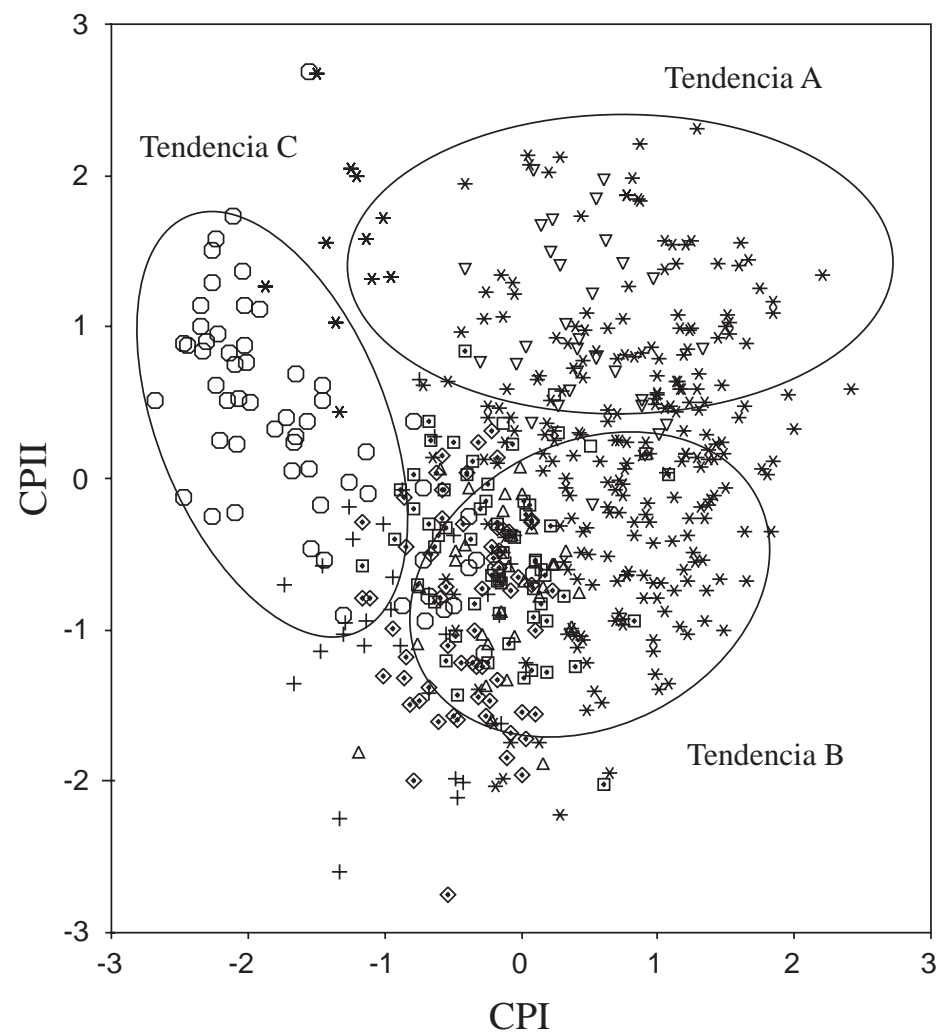

ESTADOS

- Aguascalientes

- Durango

+ Guanajuato

* J alisco

$\triangle$ Michoacán

$\nabla$ Nayarit

口 Querétaro

$\diamond$ Zacatecas

Fig. 6. Dispersión y tendencias fenotípicas observadas en la muestra, entre los componentes principales (CP) I y II.

Estas observaciones contrastan con lo conseguido por otros autores en diferentes grupos de plantas, que utilizando la misma técnica, muestran en sus resultados grupos de individuos bien segregados, los cuales son considerados como taxa diferentes (Allen, 1984; Franceschinelli et al., 1999; Henderson, 2005; Theriot y Stoermer, 1984 y Vargas, 2006).

Cuarenta por ciento de la variación morfológica analizada en Prochnyanthes permite separar plantas pequeñas de flores tubulares de plantas grandes con flores campanuladas. Sin embargo, estos fenotipos representan dos extremos de la variabilidad contenida en el género. Los patrones analizados definen una variación continua en las características morfológicas, que impiden el reconocimiento de las especies propuestas por otros autores. Asimismo, la longitud de los pedicelos no es un carácter útil, pues muestra un amplio intervalo de variación dentro de una población 
e incluso dentro de un individuo, pero no entre poblaciones. En consecuencia, $P$. mexicana es la única entidad reconocida del género, ameritando una ampliación de la descripción morfológica. Finalmente, se plantea la hipótesis de que las diferencias morfológicas observadas en las poblaciones analizadas sugieren la existencia de un proceso de especiación dentro del grupo.

Prochnyanthes mexicana (Zucc.) Rose, Contr. U.S. Nat. Herb. 8: 14. 1903. Polianthes mexicana Zucc. Abh. Math.-Phys. Cl. Königl. Bayer. Akad. Wiss. 2: 319. Prochnyanthes viridescens S. Watson, Proc. Amer. Acad. 22: 457. 1887. Prochnyanthes bulliana (Baker) Baker, Bot. Mag. 121: t. 7427. 1895. Bravoa bulliana Baker, Gard. Chron. ser. 2, 22: 328. 1884.

Hierba erecta, perenne, que emerge de un cormo vertical el cual produce raíces fusiformes carnosas; hojas (1)2-3(7), las más largas en una roseta basal, a medida que las hojas se acercan al escapo floral se hacen más pequeñas y delgadas y se confunden con las brácteas, láminas lineares a elípticas, de $8-65 \mathrm{~cm}$ de longitud y de 1-8 cm de ancho, blandas o coriáceas, verdes, ápice rematando en una espina blanda, subpecioladas, con numerosas papilas ásperas en las nervaduras y una fuerte nervadura central, bases de las hojas dilatadas, de color café, fibrosas, imbricadas, formando un bulbo delgado, fibroso y ovoide, de $8-13 \mathrm{~cm}$ de longitud; inflorescencia pseudoracemosa, escapiforme, de 0.6-2.8 $\mathrm{m}$ de altura, con 2-25 nudos florales, raquis de 20-30(-80) cm de longitud; flores dos por nudo, pedicelos de (0.02)2.5-4(-9) cm de longitud y $0.07-0.35 \mathrm{~cm}$ de diámetro, brácteas lanceoladas, de $0.5-6(-10) \mathrm{cm}$ de longitud; bractéola filiforme, de 0.1-1(-1.3) cm de longitud; perianto tubular, de 1.1-2.9 cm de longitud, de simetría bilateral, lóbulos 6 , erectos, superpuestos, deltoides, de 0.3 $1.5 \mathrm{~cm}$ de longitud y $0.3-1.2 \mathrm{~cm}$ de ancho, pubescentes en el ápice, mucho más cortos que el tubo, tubo erecto o ascendente, de $0.5-1.75 \mathrm{~cm}$ de longitud y de $0.3-0.8 \mathrm{~cm}$ de diámetro, que declina y se expande en la mitad, parte distal campanulada a tubular, de 1.1-2.5 cm de largo y 0.5-2.0 cm de ancho; estambres 6, incluidos, filamentos insertos cerca de la base de la porción expandida del tubo del perianto, de $0.8-2.0 \mathrm{~cm}$ de longitud, anteras lineares, de $0.3-1.5 \mathrm{~cm}$ de longitud y $0.1-0.7 \mathrm{~cm}$ de ancho, dorsifijas; estilo filiforme, incluido pero rebasando a los estambres, de 1.2-3.2 cm de largo y 0.6-2.2 mm de diámetro, estigma dilatado distalmente con tres lóbulos apicales, planos, recurvados y ovados, de 0.7-4.0 mm de largo; ovario ínfero, trilocular, óvulos axilares y biseriados; fruto capsular, globoso a oblongo, de $0.8-2.5 \mathrm{~cm}$ de largo y 1.0$1.7 \mathrm{~cm}$ de diámetro, trilocular, cápsula coronada por el perianto persistente; semillas planas, deltoides o semicirculares, de 0.4-0.6 cm de diámetro, negras. 


\section{AGRADECIMIENTOS}

Agradecemos a los curadores de los herbarios CIIDIR, IBUG, IEB, MEXU y QMEX las facilidades concedidas. Asimismo, gracias por las revisiones y sugerencias al manuscrito hechas por Jorge A. Pérez de la Rosa, Mollie Harker, Martha Cedano y a los revisores anónimos. A Luis Hernández se agradece la ayuda en la localización de los individuos de Querétaro. A Osvaldo Zuno agradecemos la elaboración de las ilustraciones. Por último, reconocemos la valiosa ayuda de todas las personas involucradas en el trabajo de campo.

\section{LITERATURA CITADA}

Allen, G. A. 1984. Morphological and cytological variation in the Western North America Aster occidentalis complex (Asteraceae). Syst. Bot. 9: 175-191.

Anónimo. 1999. Statistical Package for the Social Sciences (SPSS), Base 9.0. Applications Guide. Chicago, EUA.

Baker, J. G. 1884. Bravoa bulliana Baker n. sp. Gard. Chron. 22: 328.

Baker, J. G. 1895. Prochnyanthes bulliana. Bot. Mag. 121: t. 7427.

Bogler, D. J., J. L. Neff y B. B. Simpson. 1995. Multiple origins of the yucca-yucca moth association. Proc. Nat. Acad. Sci. USA 92: 6864-6867.

Bogler, D. J. y B. B. Simpson. 1995. A chloroplast DNA study of the Agavaceae. Syst. Bot. 20: 191-205.

Bogler, D. J. y B. B. Simpson. 1996. Phylogeny of Agavaceae based on its rDNA sequence variation. Am. J. Bot. 83: 1225-1235.

Clary, K. H. y B. B. Simpson. 1995. Systematics and character evolution of the genus Yucca L. (Agavaceae): evidence from morphology and molecular analyses. Bol. Soc. Bot. Mex. 56: 77-88.

Crisci, J. V. y M. F. López-Armengol. 1983. Introducción a la teoría y práctica de la taxonomía numérica. Departamento de Asuntos Científicos y Tecnológicos, Secretaría General de la Organización de los Estados Americanos. Washington, D. C. pp. 71-82.

Espejo-Serna, A. y A. R. López-Ferrari. 1993. Las monocotiledoneas mexicanas: una sinopsis florística. Lista de referencia I: Agavaceae, Alismataceae, Alliaceae, Alstroemeriaceae y Amaryllidaceae. Consejo Nacional de la Flora de México, Universidad Autónoma Metropolitana-Iztapalapa, Comisión Nacional para el Conocimiento y Uso de la Biodiversidad. México, D.F. 78 pp.

Franceschinelli, E. V., K. Yakamoto y G. J. Shepherd. 1999. Distinctions among three Simarouba species. Syst. Bot. 23: 479-488.

García-Mendoza, A. y R. Galván. 1995. Riqueza de las familias Agavaceae y Nolinaceae en México. Bol. Soc. Bot. Mex. 56: 7-24. 
Hair, J. F. Jr., R. E. Anderson, R. L. Tathan y W. C. Black. 1999. Análisis multivariante. Prentice Hall. Quinta edición. Madrid. 832 pp.

Henderson, A. 2005. A multivariate study of Calyptrogyne (Palmae). Syst. Bot. 30: 60-83.

Hernández, L. 1995. Análisis cladístico de la familia Agavaceae. Bol. Soc. Bot. Mex. 56: 57-68.

Lamboy, W. F. 1990. Disjoint Principal Component Analysis: a statistical method of botanical identification. Syst. Bot. 51: 3-12.

McVaugh, R. 1989. Bromeliaceae to Dioscoreaceae. Flora Novo-Galiciana: a descriptive account of the vascular plants of Western Mexico Vol. 15. University at Michigan Herbarium. Ann Arbor, Michigan. 398 pp.

Rose, J. N. 1903. Prochnyanthes and its species. Contr. U. S. Natl. Herb. 8(1): 13-14.

Theriot, E. y E. F. Stoermer. 1984. Principal component analysis of variation in Stephanodiscus rotula y S. niagarae (Bacillariophyceae). Syst. Bot. 9: 53-59.

Thiede, J. y U. Eggli. 1999. Einbeziehung von Manfreda Salisbury, Polianthes Linné und Prochnyanthes S. Watson in Agave Linné (Agavaceae). Kakt. and Sukk. 50: 109113.

Torres M., J. P. 2000. La utilización de técnicas estadísticas multivariables. Estudio de caso. Tesis de maestría. Centro Universitario de Ciencias Biológicas y Agropecuarias, Universidad de Guadalajara. Guadalajara, Jalisco. 102 pp.

Vargas, A. G. 2006. Variación morfológica foliar de Dioon (Zamiaceae) en la vertiente del Pacífico. Tesis de maestría. Centro Universitario de Ciencias Biológicas y Agropecuarias, Universidad de Guadalajara. Guadalajara, Jalisco. 202 pp.

Verhoek-Williams, S. E. 1975. A study of the tribe Poliantheae (including Manfreda) and revision of Manfreda and Prochnyanthes (Agavaceae). PhD thesis, Cornell University. Ithaca, New York. 405 pp.

Verhoek, S. 1978. Huaco and amole: a survey of the uses of Manfreda and Prochnyanthes. Econ. Bot. 32: 124-130.

Watson, S. 1887. List of plants collected by Dr. Edward Palmer in the state of Jalisco, Mexico in 1886. Proc. Amer. Acad. Arts 22: 396-465.

Williams, S. 1998. Agavaceae. In. Kubitzki, K. (ed.). The families and genera of vascular plants III: flowering plants. Monocotyledons, Lilianae (except Orchidaceae). Springer. Berlin. pp. 60-70.

Zuccarini, J. G. 1837. Plantarum novarum vel minus cognitarum. Abh. Math.-Phys. Cl. Königl. Bayer. Akad. Wiss. 2: 309-380. 


\section{APÉNDICE 1}

Poblaciones analizadas

\begin{tabular}{|c|c|c|}
\hline Localidad & Colector & Abreviatura \\
\hline $\begin{array}{l}\text { Aguascalientes: San José de Gracia, } 18 \text { km W } \\
\text { of San José de Gracia , camino a La Congoja, } \\
\text { Rancho Peñón Blanco, alt. } 2299 \mathrm{~m}, 22^{\circ} 10.254^{\prime} \mathrm{N} \text {; } \\
102^{\circ} 31.285^{\prime} \text { W }\end{array}$ & Rodríguez et al. 4467 & AGSGracia \\
\hline $\begin{array}{l}\text { Aguascalientes: San José de Gracia, Área Natural } \\
\text { Protegida Sierra Fría, km } 42-43 \text { camino entre El } \\
\text { Zepo y Sinaí, Rancho El Aldeano, alt. } 2540 \text { m, } \\
22^{\circ} 09^{\prime} 47^{\prime \prime} \text { N; } 102^{\circ} 39^{\prime} 03^{\prime \prime} \text { W }\end{array}$ & Castro et al. 271 & AGSsp \\
\hline $\begin{array}{l}\text { Durango: Durango, km } 19 \text { carr. Méx. } 40 \text { entre } \\
\text { Durango y El Salto, alt. } 2242 \text { m, 2358.641' N; } \\
104^{\circ} 47.066^{\prime} \mathrm{W}\end{array}$ & Rodríguez \& Castro 4542 & DGOsalt \\
\hline $\begin{array}{l}\text { Durango: Súchil, } 31.9 \mathrm{~km} \text { al SW de Súchil camino } \\
\text { a San Juan de Michis, } 3 \mathrm{~km} \text { al N de San Juan de } \\
\text { Michis, alt. } 2282 \mathrm{~m}, 23^{\circ} 28.001^{\prime} \mathrm{N} ; 104^{\circ} 08.788^{\prime} \mathrm{W}\end{array}$ & Rodríguez \& Castro 4527 & DGOsuch \\
\hline $\begin{array}{l}\text { Guanajuato: Santa Cruz de Juventino Rosas, } \\
24-27 \mathrm{~km} \text { al NNW of Juventino Rosas, km } 54- \\
56 \text { de la carr. Juventino Rosas-Santa Catarina- } \\
\text { Cd. de Guanajuato, alt. } 2255 \mathrm{~m}, 20^{\circ} 49.448^{\prime} \mathrm{N} \text {; } \\
101^{\circ} 02.049^{\prime} \mathrm{W}^{\mathrm{o}}\end{array}$ & Castro \& Frías 399 & GTOJ.R \\
\hline $\begin{array}{l}\text { Jalisco: Amatitán, km 22-23 carr. Méx. 15D, alt. } \\
1473 \text { m, 2048.215' N; } 100^{\circ} 46.183^{\prime} \mathrm{W}\end{array}$ & Rodríguez \& Castro 4594 & JALAmatit \\
\hline $\begin{array}{l}\text { Jalisco: Ameca, } 25-29 \mathrm{~km} \text { al W de Ameca, carr. } \\
\text { Méx. } 70 \text { hacia Mascota, alt. } 1513 \mathrm{~m}, 20^{\circ} 32.158^{\prime} \mathrm{N} \text {; } \\
104^{\circ} 15.087^{\prime} \mathrm{W}\end{array}$ & Rodríguez 4601 & JALAmec \\
\hline $\begin{array}{l}\text { Jalisco: Atenguillo, } 82-83 \mathrm{~km} \text { al SW de Ameca, } \\
\text { carr. Méx. } 70 \text { hacia Mascota, Puerto La Campana, } \\
\text { alt. } 1947 \mathrm{~m}, 20^{\circ} 22.238^{\prime} \mathrm{N} ; 104^{\circ} 35.697^{\prime} \mathrm{W}\end{array}$ & Rodríguez \& Castro 4626 & JALCampa \\
\hline $\begin{array}{l}\text { Jalisco: Tapalpa, 1-2 km camino de terracería a } \\
\text { Las Piedrotas-Los Frailes, hacia Chiquilistlán, } \\
\text { alt. } 2327 \mathrm{~m}, 20^{\circ} 00.267^{\prime} \mathrm{N} ; 103^{\circ} 48.707^{\prime} \mathrm{W}^{\mathrm{o}} \text {. }\end{array}$ & Castro et al. 312 & JALTapalp \\
\hline $\begin{array}{l}\text { Jalisco: Tlajomulco de Zúñiga, km } 11-12 \text { carr. } \\
\text { Mex. } 15 \text { Guadalajara-Morelia, justo frente a } \\
\text { las Plazas Outlet, alt. } 1624 \mathrm{~m}, 20^{\circ} 31.186^{\prime} \mathrm{N} \text {; } \\
103^{\circ} 28.984^{\prime} \mathrm{W}\end{array}$ & Castro \& Rueda 304 & JALOutlet \\
\hline
\end{tabular}


Continuación. Apéndice 1.

\begin{tabular}{|c|c|c|}
\hline Localidad & Colector & Abreviatura \\
\hline $\begin{array}{l}\text { Jalisco: Zapopan, Parque El Centinela, entre Las } \\
\text { Cañadas y Río Blanco, alt. } 1582 \text { m, } 20^{\circ} 45.660^{\prime} \mathrm{N} \text {; } \\
103^{\circ} 22.949^{\prime} \mathrm{W}\end{array}$ & Castro 775 & JALcenti \\
\hline $\begin{array}{l}\text { Jalisco: Zapopan, Fraccionamiento Pinar de la } \\
\text { Venta, km } 15 \text { carr. Mex. } 15 \text { Guadalajara-Tepic, } \\
\text { alt. } 1740 \mathrm{~m}, 20^{\circ} 43.015^{\prime} \mathrm{N} ; 103^{\circ} 31.516^{\prime} \mathrm{W}\end{array}$ & Castro 308 & JALpinar \\
\hline $\begin{array}{l}\text { Jalisco: Zapopan, Río Blanco, } 500 \text { m al E de El } \\
\text { Diente, alt. } 1564 \text { m, } 20^{\circ} 47.125^{\prime} \mathrm{N} ; 103^{\circ} 23.566^{\prime} \mathrm{W}\end{array}$ & Castro 393 & JALR.Bco \\
\hline $\begin{array}{l}\text { Jalisco: Zapopan, Bosque El Nixticuil, Lomas de } \\
\text { Zapopan, al NW del centro de Zapopan, alt. } 1620 \\
\text { m, } 20^{\circ} 44.9650^{\prime} \mathrm{N} ; 103^{\circ} 24.370^{\prime} \mathrm{W}\end{array}$ & Castro 272 & JALnixti \\
\hline $\begin{array}{l}\text { Michoacán: Peribán de Ramos, } 5 \text { km al SW de } \\
\text { Peribán de Ramos, camino a Buenavista Tomatlán, } \\
\text { alt. } 1615 \mathrm{~m}, 19^{\circ} 28.586^{\prime} \mathrm{N} ; 102^{\circ} 26.426^{\prime} \mathrm{W}\end{array}$ & Rodríguez et al. 4359 & $\mathrm{MICH}$ \\
\hline $\begin{array}{l}\text { Nayarit: Santa María del Oro, km 189-190 carr. } \\
\text { Méx. } 15,3 \mathrm{~km} \text { al E de La Galinda, alt. } 1272 \mathrm{~m} \text {, } \\
21^{\circ} 18.029^{\prime} \mathrm{N} ; 104^{\circ} 39.077^{\prime} \mathrm{W}\end{array}$ & Rodríguez \& Castro 4586 & NAYoro \\
\hline $\begin{array}{l}\text { Querétaro: Huimilpan, camino de ascenso al } \\
\text { cerro Capula, al NW of Huimilpan, alt. } 2317 \mathrm{~m} \text {, } \\
20^{\circ} 20.815^{\prime} \mathrm{N} \text {; } 100^{\circ} 17.972^{\prime} \mathrm{W}\end{array}$ & Castro \& Frías 410 & QRO \\
\hline $\begin{array}{l}\text { Zacatecas: Florencia de Benito Juárez, Rancho } \\
\text { Potrerillos, } 15 \mathrm{~km} \text { al NW de Florencia de Benito } \\
\text { Juárez, alt. } 2100 \mathrm{~m}, 21^{\circ} 32^{\prime} 13^{\prime \prime} \mathrm{N} ; 103^{\circ} 38^{\prime} 03^{\prime \prime} \mathrm{W}\end{array}$ & Castro \& Ramírez 240 & ZACFlor \\
\hline $\begin{array}{l}\text { Zacatecas: Tlaltenango de Sánchez Román, } 26.7 \\
\text { km SE of Tlaltenango, camino a Jalpa, Sierra de } \\
\text { Morones, alt. } 2340 \mathrm{~m}, 21^{\circ} 39.897^{\prime} \mathrm{N} ; 103^{\circ} 09.316^{\prime} \mathrm{W}\end{array}$ & Castro \& Rodríguez 396 & ZACMor \\
\hline
\end{tabular}




\section{APÉNDICE 2}

\section{Ejemplares examinados}

Aguascalientes: Municipio de Calvillo: sur de la presa Los Alamitos, $3 \mathrm{~km}$ al E de Los Alisos, G. García 4314 (CIIDIR); Sierra del Laurel, cerca del límite Aguascalientes-Jalisco, 10 millas al SE de El Calvillo (3 hrs a caballo del R. Los Adobes), R. McVaugh 18442 (IEB, MEXU). Municipio de San José de Gracia: Ojo de Agua El Durazno, G. García 2487 (CIIDIR); 13 km después del Cepo, $3 \mathrm{~km}$ antes del Sinaí, carretera San José de Gracia-La Labor, A. R. López-Ferrari et al. 2259 (IEB, MEXU). Durango: Municipio de Durango: route 40 just past turnoff to Tepalcates, W de $53 \mathrm{~km}$ marker, R. R. Clinebell II 3016 (CIIDIR); predio Las Bayas, Universidad Juárez del Estado de Durango, S. González \& A. García 4576 (CIIDIR); predio las Bayas, Universidad Juárez del Estado de Durango, S. González \& A. García 4582 (IEB); ejido Regocijo Manantial, R. Carrillo 267 (CIIDIR); ejido San José de Ánimas, Cordón del Indio, A. García 4068 (CIIDIR); San Dimas, ejido Vencedores, $\mathrm{S}$ del poblado Vencedores rodal 1273, A. Park 174 (CIIDIR); $5.3 \mathrm{~km}$ al W del ejido Los Mimbres, H. H. Iltis et al. 209 (IBUG, MEXU); Pueblo Nuevo, carretera Dgo.Mazatlán, área semillera UAFG, aprox. km 10, A. Benítez S-43 (CIIDIR); carretera Dgo.-La Flor, a la altura de La Mona, A. García et al. 1057 (CIIDIR); ca. 7 km al E de Navíos, carretera Durango-El Salto, A. R. López-Ferrari et al. 3009 (CIIDIR). Municipio de Súchil: Toribia, Reserva la Michilía, M. E. Maury, et al. 67 (IEB); San Juan de Michis, cerca del arroyo de Raymundo, S. Acevedo 122 (CIIDIR); San Juan de Michis al S del poblado, Reserva de la Biosfera La Michilía, M. F. Passini 19482 (CIIDIR); El Temazcal, 4 km al S de Piedra Herrada (La Michilía), J. Rzedowski 37501 (CIIDIR, IEB); Cañada Nana Juana, Rancho La Cebolla, S. González 3197 (CIIDIR); San Juan de Michis, 5 km reserva La Michilía, S. González 3859 (CIIDIR); La Corraleña entre San Juan de Michis y Piedra Herrada, Reserva de la Biosfera La Michilía, S. González 4730 (IEB, CIIDIR). Municipio de Mezquital: 3 km al W de Santa María Ocotán, M. González 1400 (CIIDIR); 16 km de el Durazno por el camino a los Charcos, M. González \& R. Galván 2021 (CIIDIR); alrededores de La Guajolota, I. Solís 233 (CIIDIR); ciénega del Coyote, aproximadamente 15 km al SE de Santa María Ocotán, I. Solís 137 (IBUG). Guanajuato: Municipio de Juventino Rosas: 24 km al NNW de Juventino Rosas, S. Zamudio 5683 (IEB); 23 km al NNW de Juventino Rosas, J. Rzedowski 53591 (IEB, MEXU). Municipio de Ocampo: $12 \mathrm{~km}$ al S de Ibarra, sobre la carretera a León, J. Rzedowski 52268 (IEB); 4-5 km al S de Santa Bárbara, E. Pérez \& E. Carranza 3126 (IEB). Municipio de 
Pénjamo: 15 km al E de Tierras Negras, E. Carranza 4271 (IEB). Municipio de San Felipe: La Atarjea, S. Zamudio 4074 (IEB). Jalisco: Municipio de Bolaños: 20 km antes de Tezompa por la brecha de Bolaños-Huejuquilla el Alto, H. Arreola \& L. Guzmán 504 (IBUG); Escobas, 18 km al NW de San Martín de Bolaños, A. FloresMacías 1249 (IBUG); Sierra de Bolaños, El Platanar Yerbanis, L. M. Villarreal de Puga 1905 (IBUG). Municipio de Colotlán: puente la Cantera al O de Temastián, J. A. Machuca \& M. Ibarra 8082 (IEB). Municipio de Ejutla: cima del cerro localizado al SW de Ejutla, junto al cerro El Narigón, carretera Ejutla-El Grullo, A. Rodríguez \& A. Castro-Castro 4000 (IBUG, MEXU). Municipio de Guachinango: km 51 carretera Ameca-Mascota, A. Rodríguez \& L. Ortiz-Catedral 3193 (IBUG). Municipio de Guadalajara: brecha a San Isidro Mazatepec, por la prolongación Mariano Otero, km 16, O. Reyna 514 (IBUG). Municipio de Ixtlahuacán del Río: Ixtlahuacán del Río, S. Guerrero \& M. Cházaro 278 (IEB). Municipio de Jocotepec: Cerro Viejo subiendo por Las Trojes, M. Cházaro et al. 5025 (IBUG, IEB). Municipio de Magdalena: Rancho de San Simón y San Matías, al NO de Magdalena, A. Sánchez-Gutiérrez 37 (IBUG). Municipio de Mascota: km 8 camino Juanacatlán-El Saucillo, O. Reyna \& S. Pérez 697 (IBUG). Municipio de Mazamitla: $10 \mathrm{~km}$ al NO de La Central, brecha a Corral de Mejía, J. Villa \& J. Chávez 483 (IBUG, IEB). Municipio de Mezquitic: $4 \mathrm{~km}$ al NE de Pinos Altos por la brecha a Tezompa, F. J. Santana-Michel 1824 (IBUG); km 13 carretera Monte Escobedo-Mezquitic, R. Ramírez-Delgadillo \& $R$. González-Tamayo 1440 (IBUG). Municipio de Poncitlán: Casa Blanca, L. M. Villarreal de Puga 16313 (IBUG). Municipio de Talpa de Allende: Sierra de Cuale, SO de Talpa de Allende, SO de Piedra Rajada, $R$. McVaugh 14266 (IEB); 4-5 millas al NNE de Talpa de Allende, R. McVaugh 20369 (IEB). Municipio de Tapalpa: camino a las Piedras Bolas, $2 \mathrm{~km}$ al NO de Tapalpa, A. Flores \& R. Cuevas 530 (IBUG); Sierra de Tapalpa, $3 \mathrm{~km}$ al NE de Tapalpa por el camino a la Laguna de Sayula H. H. Iltis 20172 (IBUG); camino a Sayula, Cerro de Talcozagua, H. H. Iltis 708 (IBUG). Municipio de Tenamaxtlán: Los Picachos, J. A. Machuca \& M. Cházaro 7929 (IEB). Municipio de Tequila: Volcán de Tequila, $8.4 \mathrm{~km}$ sobre la brecha a la cima, A. R. López-Ferrari et al. 2390 (IEB); Volcán de Tequila, L. M. Villarreal de Puga 14119 (IBUG); 2 millas al NO de Tequila, R. McVaugh 18621 (IEB). Municipio de Tlajomulco de Zúñiga: brecha a Los Ocotes, $5 \mathrm{~km}$ al E de Nicolás R. Casillas, $L$. Ortiz-Catedral 105 (IBUG); Cerro Viejo, ladera de exposición N frente a San Miguel Cuyutlán, J. A. Machuca 3281 (IEB). Municipio de Zapopan: km 14 carretera a San Cristóbal de la Barranca, R. Ornelas 585 (IBUG); cerro El Colli, G. González et al. 4 (IBUG); Los Robles-San Patricio, A. García \& D. Álvarez 56 (IBUG); cerro del hospital Ángel Leaño, M. Cházaro \& J. Cortés 7742 (IEB); Mesa Colorada, A. 
Rodríguez \& J. Suárez 1455 (IBUG); Mesa Colorada, A. Rodríguez 1454 (IEB); mirador de la barranca, km 21 carretera a Colotlán, P. Carrillo-Reyes \& M. Cházaro 458 (IBUG); Santa Cruz del Astillero, R. Hernández-Magaña et al. 9539 (CIIDIR); bosque La Primavera, L. M. Villarreal de Puga 11466 (IBUG). Michoacán: Municipio de Coeneo: base del cerro La Calabaza, cerca de la desviación a Coeneo, H. Díaz-Barriga 2550 (CIIDIR, IBUG, IEB, MEXU). Municipio de Lagunillas: La Caja, J. M. Escobedo 2065 (IEB). Municipio de Paracho: 5 km al SW de Paracho, M. Pérez 138 (CIIDIR, IEB). Municipio de Quiroga: cerro Tzirate, C. López 1138 (IEB). Municipio de Villa Jiménez: cerro El Brinco, E. Pérez \& E. García 1531 (IEB). Municipio de Ziracuaretiro: 4 km al S de San Andrés Coru, S. Zamudio 11532 (IEB). Municipio de Nahuatzen: al S de Arentepacua, E. García \& E. Pérez 2975 (IEB). Nayarit: Municipio de Ahuacatlán: 10 millas al SE de Ahuacatlán, camino a la Barranca del Oro, C. Feddema 342 (IEB). Municipio de La Yesca: rancho Los Planes, camino a San Pelayo, A. Flores 2262 (IBUG, IEB). Municipio de Santa María del Oro: km 189-190 carretera Guadalajara-Tepic, entre Jazmines y la Galinda, $A$. Rodríguez et al. 3237 (IBUG). Municipio de Xalisco: estación de microondas Cerro Boludo, O. Téllez-Valdés et al. 13024 (IBUG). Querétaro: Municipio de Huimilpan: cerro Capula, $1 \mathrm{~km}$ al N de la carretera a San Pedro Huimilpan, L. Hernández 4261, 4267 (IEB, MEXU, QMEX). Zacatecas: Municipio de Juchipila: cerro El Piñón, parte alta de Los Patos (Sierra de Morones) Pueblo Viejo, J. A. Lomelí \& S. Carvajal 2525 (IEB); Municipio de Nochixtlán: El Capulín, R. Pérez-Pérez s.n. (IBUG). 
